\title{
A growth gap for diffeomorphisms of the interval
}

\author{
Leonid Polterovich and Mikhail Sodin \\ School of Mathematical Sciences \\ Tel Aviv University \\ Ramat Aviv, Israel 69978 \\ polterov@post.tau.ac.il_sodin@post.tau.ac.il
}

\begin{abstract}
Given an orientation-preserving diffeomorphism of the interval $[0 ; 1]$, consider the uniform norm of the differential of its $n$-th iteration. We get a function of $n$ called the growth sequence. Its asymptotic behaviour is an interesting invariant which naturally appears both in geometry of the diffeomorphisms groups and in smooth dynamics. Our main result is the following Gap Theorem: the growth rate of this sequence is either exponential, or at most quadratic with $n$. Further, we construct diffeomorphisms with quite an irregular behaviour of the growth sequence. This construction easily extends to arbitrary manifolds.
\end{abstract}

\section{Introduction and main results}

Denote by $\operatorname{Diff}_{0}([0 ; 1])$ the group of all $C^{1}$-diffeomorphisms $f$ of the interval $[0 ; 1]$ such that $f(0)=0$ and $f(1)=1$. Given a diffeomorphism $f \in \operatorname{Diff}_{0}([0 ; 1])$, define its growth sequence

$$
\Gamma_{n}(f)=\exp \left\|\log \left(f^{n}\right)^{\prime}\right\|_{\infty}=\max \left(\left\|\left(f^{n}\right)^{\prime}\right\|_{\infty},\left\|\left(f^{-n}\right)^{\prime}\right\|_{\infty}\right), \quad n \in \mathbb{N} .
$$

Here $\|.\|_{\infty}$ stands for the uniform norm, and $f^{n}, n \in \mathbb{Z}$, denotes the $n$ th iterate of $f$. Let us say that two sequences of positive real numbers are 
equivalent if their ratio is bounded away from 0 and $+\infty$. The equivalence class of the sequence $\Gamma_{n}(f)$ is called the growth type of $f$. Clearly, it is invariant under conjugations in the group of diffeomorphisms.

From the viewpoint of dynamics, the growth type reflects asymptotic distortion of length under iterations of $f$. Geometrically, the growth type of $f$ is closely related to the distortion of the cyclic subgroup $\left\{f^{n}\right\} \subset \operatorname{Diff}_{0}([0 ; 1])$ with respect to the multiplicative norm $\Gamma_{1}(f)$ on $\operatorname{Diff}_{0}([0 ; 1])$. In [DG] D'Ambra and Gromov suggested to study the growth type for various classes of diffeomorphisms.

The growth sequence is always submultiplicative:

$$
\Gamma_{n+m}(f) \leq \Gamma_{n}(f) \Gamma_{m}(f),
$$

therefore there always exists the limit

$$
\gamma(f)=\lim _{n \rightarrow \infty} \Gamma_{n}^{1 / n}(f) .
$$

Let $\operatorname{Fix}(f)$ be the set of fixed points of $f$. Using standard arguments of ergodic theory, it is easy to check that

$$
\gamma(f)=1 \quad \text { if and only if } \quad f^{\prime}(\xi)=1 \quad \text { for every } \xi \in \operatorname{Fix}(f)
$$

(we bring details in the end of Section 2). Otherwise, $\gamma(f)>1$, so $\Gamma_{n}(f)$ grows exponentially fast. Loosely speaking, the exponent $\gamma(f)$ distinguishes between the parabolic and hyperbolic behaviour of diffeomorphisms. Our main result establishes the growth gap between the parabolic and hyperbolic cases.

Theorem 1.3 (Growth gap) Let $f \in$ Diff $_{0}([0 ; 1])$ be a $C^{2}$-diffeomorphism with $\gamma(f)=1$. Then

$$
\Gamma_{n}(f) \leq \text { Const } n^{2}
$$

for every $n \in \mathbb{N}$. 
As the proof shows, the $C^{2}$-condition can be relaxed by assuming that $\log f^{\prime}$ has bounded variation on $[0 ; 1]$. Probably it cannot be relaxed much further. Under the assumptions of the theorem, the estimate is sharp. For instance, if $f \in \operatorname{Diff}_{0}([0 ; 1])$ is a $C^{\infty}$-diffeomorphism such that $\operatorname{Fix}(f)=$ $\{0,1\}, f^{\prime}(0)=f^{\prime}(1)=1$ but $f^{\prime \prime}(0) \neq 0, f^{\prime \prime}(1) \neq 0$, then one can check that the growth type of $f$ is $n^{2}$.

The result can be considered in the following more general context. Let $G$ be a group endowed with a multiplicative (pseudo)-norm $\rho$, that is a function $\rho: G \rightarrow[0 ;+\infty)$ satisfying $\rho(\mathbb{1})=1, \rho(f)=\rho\left(f^{-1}\right)$, and $\rho(f g) \leq \rho(f) \rho(g)$. By a growth gap we mean a gap in the possible growth types of sequences $\rho\left(g^{n}\right)$ for $g \in G$. Existence of growth gaps is known for finite-dimensional Lie groups. As a toy model, consider the group $G L(m, \mathbb{R})$ endowed with the operator norm. For instance, when $m=2$ the possible growth types are given by $e^{c n} n^{q}$ where $c \geq 0, q \in\{0,1\}$. Other examples of growth gaps are given by certain discrete groups endowed with the norm $e^{l(w)}$, where $l(w)$ is the word length of an element $w$ with respect to a chosen set of generators. See [LMR] for the treatment of lattices. As far as we know, Theorem 1.3 gives the first example of a growth gap for an infinite-dimensional Lie group (though see [PSib] for some steps in this direction in the context of Hofer's metric on groups of area-preserving diffeomorphisms).

Our second result starts with another observation:

$$
\sum_{n \geq 1} \frac{1}{\Gamma_{n}(f)}<\infty \quad \text { for every } f \in \operatorname{Diff}_{0}([0 ; 1]) \backslash\{\mathbb{1}\} .
$$

Indeed, take a point $x_{0} \in[0 ; 1] \backslash \operatorname{Fix}(f)$ and assume, for example, that $f\left(x_{0}\right)>$ $x_{0}$. Put $x_{n}=f^{n} x_{0}, \delta_{n}=x_{n+1}-x_{n}$. Note that $\left[x_{0} ; x_{1}\right]=f^{-n}\left[x_{n} ; x_{n+1}\right]$, so

$$
\Gamma_{n}(f) \geq \max _{x}\left(f^{-n}\right)^{\prime}(x) \geq \delta_{0} / \delta_{n}
$$

Obviously, $\sum_{n \in \mathbb{Z}} \delta_{n} \leq 1$ and thus (1.4) follows. In particular, we see that

$$
\Gamma_{n}(f) \geq \operatorname{const} n
$$


for "most" indices $n \in \mathbb{N}$. In many cases, (1.6) holds for all $n \in \mathbb{N}$, see a brief discussion below. However, the next theorem shows that there are nontrivial $C^{\infty}$-diffeomorphisms with an arbitrary slow growth of $\Gamma_{n}(f)$ along a rare subsequence of indices $n$.

Denote by $\mathcal{L}$ the set of all strictly increasing sequences $\{u(n)\}, n \in \mathbb{N}$, of positive real numbers with $u(n) \rightarrow+\infty$ as $n \rightarrow+\infty$.

Theorem 1.7 For every sequence $u \in \mathcal{L}$ there exists a diffeomorphism $f$ in $\operatorname{Diff}_{0}([0 ; 1]) \backslash\{\mathbb{1}\}$ such that

$$
\liminf _{n \rightarrow \infty} \frac{\Gamma_{n}(f)}{u(n)} \leq 1
$$

The diffeomorphism we construct in Theorem 1.7 must oscillate near the end points. Consider the function $v(x)=f(x)-x$. Assume for a moment that $v$ is monotone near $x=1$. After appropriate choice of $x_{0}$ we can think that $v$ is non-increasing on $\left[x_{0} ; 1\right]$. Then the sequence $\delta_{n}=f^{n+1} x_{0}-f^{n} x_{0}$, $n \geq 1$, is monotone as well. Thus $\delta_{n} \leq \frac{1}{n}$ since $\sum \delta_{n} \leq 1$. Therefore, by (1.5), $f$ satisfies (1.6) for all indices $n \in \mathbb{N}$. Let us say that a diffeomorphism $f$ is flat at the end points if $f^{\prime}(p)=1, f^{(i)}(p)=0$ for $p \in\{0 ; 1\}$ and all integers $i \geq 2$. If $f$ is not flat near 1 , then the Taylor expansion of $f$ at the point $x=1$ yields monotonicity of the function $v(x)$ for $x$ sufficiently close to 1 , and therefore at least linear growth of the sequence $\Gamma_{n}(f)$. Hence the diffeomorphism from Theorem [1.7 must be a flat one. In what follows, we will design an oscillating function $v$ which forces $f$ to satisfy $\Gamma_{n_{i}}(f)=o\left(n_{i}\right)$, $\left\{n_{i}\right\} \subset \mathbb{N}$. Of course these oscillations are rare and small since $\Sigma \delta_{n}$ converges. An additional difficulty is that they have to be not too steep since we wish $f$ to be $C^{\infty}$-smooth. Let us mention that flat diffeomorphisms of $[0 ; 1]$ with an oscillating $v$ were considered in a different context in the papers $[\mathrm{Se}], \mathrm{K}]$.

Theorem 1.7 has a straightforward corollary pertaining to diffeomorphisms of arbitrary compact manifolds $M$. Let $\operatorname{Diff}_{0}(M)$ be the group of all $C^{1}$-smooth diffeomorphisms isotopic to the identity map 1 . Given a dif- 
feomorphism $f \in \operatorname{Diff}_{0}(M)$, define as above its growth sequence

$$
\Gamma_{n}(f)=\max \left(\max _{x \in M}\left\|d_{x} f^{n}\right\|, \max _{x \in M}\left\|d_{x} f^{-n}\right\|\right), \quad n \in \mathbb{N} .
$$

Here $\left\|d_{x} f\right\|$ stands for the operator norm of the differential $d_{x} f$ calculated with respect to a Riemannian metric on $M$.

Corollary 1.8 Let $B$ be a closed Euclidean ball of dimension $\geq 1$. For every $u \in \mathcal{L}$ there exists a $C^{\infty}$-diffeomorphism $g \in$ Diff $(B) \backslash\{11\}$ which equals the identity near the boundary and whose growth sequence satisfies

$$
\liminf _{n \rightarrow+\infty} \frac{\Gamma_{n}(g)}{u(n)} \leq 1 \quad \text { and } \quad \sum_{n=1}^{\infty} \frac{1}{\Gamma_{n}(g)}<\infty .
$$

Proof: Let $f$ be a diffeomorphism of $\left[\frac{1}{3} ; \frac{2}{3}\right]$ which is flat at the end points and satisfies $\liminf _{n \rightarrow+\infty} \frac{\Gamma_{n}(f)}{u(n)} \leq 1$. Existence of such diffeomorphism follows from Theorem [1.7 and discussion above. Extend $f$ by the identity map to the whole interval $[0 ; 1]$. We get a smooth diffeomorphism $h$ of $[0 ; 1]$ which satisfies (1.9). Define now a diffeomorphism $g$ of the ball $B=\{|x| \leq 1\}$ by $g(x)=x h(|x|) /|x|$. Clearly, $g$ equals the identity outside the spherical annulus $A=\left\{\frac{1}{3} \leq|x| \leq \frac{2}{3}\right\}$. We claim that $g$ also satisfies conditions (1.9). Indeed, $g^{n}(x)=x h^{n}(|x|) /|x|$ for every $n \in \mathbb{Z}$. Take a tangent vector $v \in T_{x} \mathbb{R}^{m}$ and decompose it as $v=\xi+\eta$, where $\xi$ is parallel to $x$ and $\eta$ is orthogonal to $x$. One readily calculates that

$$
d_{x} g(v)=\left(h^{n}\right)^{\prime}(|x|) \xi+\frac{h^{n}(|x|)}{|x|} \eta
$$

for $x \in B \backslash\{0\}$. Note that $|x|^{-1} h^{n}(|x|) \in\left[\frac{1}{2} ; 2\right]$ for all $x \in A$. This yields

$$
\Gamma_{n}(h) \leq \Gamma_{n}(g) \leq \max \left(2, \Gamma_{n}(h)\right)
$$

Since $\Gamma_{n}(h) \rightarrow \infty$ in view of (1.4), we see that $\Gamma_{n}(g)=\Gamma_{n}(h)$ for large $n$. Hence $g$ also satisfies conditions (1.9). 
Remark. Corollary 1.8 immediately extends to an arbitrary compact manifold. Indeed, fix a closed ball inside the manifold and extend the diffeomorphism $g$ constructed in the theorem by the identity map. We get a diffeomorphism from $\operatorname{Diff}_{0}(M) \backslash\{\mathbb{1}\}$ which satisfies (1.9).

It is interesting to compare this remark with restrictions on the growth type of symplectic maps which were obtained recently in [P1], [PS]. For instance, let $f$ be an area-preserving $C^{\infty}$-diffeomorphism of the 2-dimensional torus which is isotopic to the identity map $\mathbb{1}$. Assume that $f \neq \mathbb{1}$ and $f$ has a fixed point. Then according to [PS] the growth type of $f$ is at least linear, that is, (1.6) holds for all indices $n \in \mathbb{N}$. We refer to [P1] for extensions to other symplectic manifolds including all closed surfaces of higher genus ${ }^{1}$ and for further discussion. Clearly the fixed point condition is crucial there. Indeed, if $f$ is a translation of the torus, the sequence $\Gamma_{n}(f)$ is bounded (see [P2 for more sophisticated examples). On the other hand, as we have seen above, there exists a $C^{\infty}$-diffeomorphism which has fixed points but violates inequality (1.6) for a subsequence.

\section{An Outlook}

Flat fixed points form a major difficulty in the study of the growth for $C^{\infty}$ diffeomorphisms of the interval. One can speculate that further understanding of their influence on the growth sequence leads to a rather satisfactory description of the "spectrum" of possible growth types. The Gap Theorem and the examples provided by Theorem [1.7 correspond to the opposite ends of this spectrum. To be more precise recall that if a diffeomorphism $f$ has a non-degenerate fixed point $\xi$ (that is $f^{\prime}(\xi) \neq 1$ ) its growth is exponential. Assume now that all fixed points of $f$ are degenerate but non-flat. We say that $\xi \in \operatorname{Fix}(f)$ has order $p \in \mathbb{N}$ if $f^{(j)}(\xi)=0$ for all $j=2, \ldots, p$ but

$f^{(p+1)}(\xi) \neq 0$. In this case one should be able to show (using e.g. the Takens

\footnotetext{
${ }^{1} \mathrm{P}$. LeCalvez informed us that he can prove this result for surfaces by a different method.
} 
normal form [T] that

$$
\Gamma_{n}(f) \sim n^{\frac{p+1}{p}},
$$

where $p$ is the minimal order of the fixed points of $f$. Therefore, in the general case, we arrive at the following problem: What is the contribution of flat fixed points to the growth type of $f$ ? Warning: setting $p=\infty$ in formula (1.10) leads to the answer $\Gamma_{n} \sim n$ which is obviously wrong: it contradicts (1.4). In fact, Theorem [1.7 shows that flat fixed points sometimes yield an irregular behavior of the growth sequence. Nevertheless an optimistic scenario is that the contribution of flat fixed points does not exceed $n^{1+\epsilon}$ for every $\epsilon>0$. Note that the Gap Theorem confirms this for $\epsilon=1$. If this is indeed true, we get an infinite sequence of new gaps formed by the growth types

$$
\left\{n^{\frac{p+1}{p}}\right\}, \quad p \in \mathbb{N} \text {. }
$$

This picture, though quite an enticing, at the moment seems to be out of reach. Our proof of the Gap Theorem goes in another direction and completely ignores higher derivatives at the fixed points (as a compensation, we work in the $C^{2}$-category). Let us conclude this discussion with the following test

Question 1.11 Suppose that $f$ is a sufficiently smooth diffeomorphism of $[0 ; 1]$ with Fix $(f)=\{0 ; 1\}$. Assume that $f^{\prime}(0)=f^{\prime}(1)=1$ and $f^{\prime \prime}(0)=$ $f^{\prime \prime}(1)=0$. Is it true that $\Gamma_{n}(f)=o\left(n^{2}\right)$ as $n \rightarrow \infty$ ?

Added on March, 2003. In a recent preprint [B, A. Borichev confirms formula (1.10) and gives the affirmative answer to Question 1.11. At the same time, according to [B] our "optimistic scenario" appeared to be wrong.

\section{Existence of the growth gap}

In this section we prove Theorem 1.3 and check relation (1.2). 
Let $f$ be a $C^{2}$-diffeomorphism of $[0 ; 1]$ with $\gamma(f)=1$. Let $v(f)$ be the variation of $\log f^{\prime}$ on the interval $[0 ; 1]$. We shall use a classical

Lemma 2.1 (Denjoy) If $J \subset[0 ; 1]$ is a closed interval such that $f J \cap J=\emptyset$, then for every $n \in \mathbb{N}$ and every $x, y \in J$

$$
e^{-v(f)} \leq \frac{\left(f^{n}\right)^{\prime}(x)}{\left(f^{n}\right)^{\prime}(y)} \leq e^{v(f)} .
$$

For $n \geq 0$ put

$$
a_{n}(f)=\max _{[0 ; 1]} \log \left(f^{n}\right)^{\prime}(x) \quad \text { and } \quad a_{n}\left(f^{-1}\right)=\max _{[0 ; 1]} \log \left(f^{-n}\right)^{\prime}(x) .
$$

Note that $a_{0}\left(f^{ \pm}\right)=0$. These two sequences appear to be "almost convex":

Lemma 2.2 The sequences $a_{n}=a_{n}(f)$ (resp. $a_{n}=a_{n}\left(f^{-1}\right)$ ) satisfy the inequality

$$
2 a_{n}-a_{n-1}-a_{n+1} \leq C(f) e^{-a_{n}}, \quad n \in \mathbb{N},
$$

with $C(f)=L(f) e^{v(f)}$, where $L(f)$ is the Lipschitz constant of the function $\log f^{\prime}$.

Proof of Lemma 2.1; We prove the statement for the sequence $a_{n}=a_{n}(f)$. The proof for the second sequence is the same (note that $v(f)=v\left(f^{-1}\right)$ ). Choose $x_{0}$ such that $a_{n}=\log \left(f^{n}\right)^{\prime}\left(x_{0}\right)$. In view of (1.2) $x_{0}$ does not belong to $\operatorname{Fix}(f)$. Put $x_{j}=f^{j} x_{0}, j \in \mathbb{Z}$. Then we have

$$
\begin{gathered}
a_{n+1} \geq \log \left(f^{n+1}\right)^{\prime}\left(x_{-1}\right)=\sum_{j=-1}^{n-1} \log f^{\prime}\left(x_{j}\right), \\
a_{n-1} \geq \log \left(f^{n-1}\right)^{\prime}\left(x_{1}\right)=\sum_{j=1}^{n-1} \log f^{\prime}\left(x_{j}\right),
\end{gathered}
$$

and

$$
a_{n}=\sum_{j=0}^{n-1} \log f^{\prime}\left(x_{j}\right)
$$


Therefore,

$$
\begin{aligned}
2 a_{n}-a_{n-1}-a_{n+1} & \leq \log f^{\prime}\left(x_{0}\right)-\log f^{\prime}\left(x_{-1}\right) \\
& \leq L(f)\left|x_{0}-x_{-1}\right|<L(f) \frac{\left|x_{0}-x_{-1}\right|}{\left|x_{n}-x_{n-1}\right|} \\
& =\frac{L(f)}{\left(f^{n}\right)^{\prime}(y)} \quad\left(y \in\left(x_{-1}, x_{0}\right)\right) \\
& \leq \frac{L(f) e^{v(f)}}{\left(f^{n}\right)^{\prime}\left(x_{0}\right)}=C(f) e^{-a_{n}},
\end{aligned}
$$

In the last inequality, we apply Lemma 2.1 to the interval $\left[y ; x_{0}\right]$. We are done.

The next lemma is crucial:

Lemma 2.3 (Growth lemma) Let $\left\{a_{n}\right\}_{n \geq 1}$ be a sequence of real numbers such that for each $n \geq 1$

$$
2 a_{n}-a_{n-1}-a_{n+1} \leq C e^{-a_{n}}, \quad C>0
$$

and $a_{0}=0$. Then either for each $n \geq 1$

$$
a_{n} \leq 2 \log \left(n \sqrt{\frac{C}{2}}+1\right)
$$

or

$$
\liminf _{n \rightarrow \infty} \frac{a_{n}}{n}>0
$$

Proof of Theorem 1.3; Since $\Gamma_{n}(f)=\exp \left(\max \left(a_{n}(f), a_{n}\left(f^{-1}\right)\right)\right.$, Lemmas 2.2 and 2.3 yield Theorem 1.3

Proof of Lemma 2.3; Introduce the second difference operator

$$
L_{j} p=2 p_{j}-p_{j-1}-p_{j+1}, \quad j \geq 1
$$


acting on sequences $\left\{p_{j}\right\}, j \geq 0$. Set $D=\sqrt{\frac{C}{2}}$ and observe that the sequence $h_{j}=2 \log (j \sqrt{C / 2}+1)$ is a super-solution of the non-linear second order difference equation

$$
L_{j} p=C e^{-p_{j}}
$$

Indeed

$$
L_{j} h=2 \log \frac{(D j+1)^{2}}{(D j+1)^{2}-D^{2}}>2 \frac{D^{2}}{(D j+1)^{2}}=C e^{-h_{j}} .
$$

Here we used inequality $\log (A / B)>(A-B) / A$ valid for $A>B>0$. On the other hand condition (2.4) says that the sequence $\left\{a_{j}\right\}$ is a sub-solution of the same equation (2.7) with $a_{0}=h_{0}=0$. Our first claim is that for any $\epsilon \geq 0$ the sequence $b_{j}=a_{j}-(1+\epsilon) h_{j}$ has no positive local maxima. This is a version of the maximum principle for equation (2.7). Indeed, suppose that $i \geq 1$ is a local maximum of $\left\{b_{j}\right\}$. Then $L_{i} b \geq 0$, and

$$
C e^{-a_{i}} \geq L_{i} a=L_{i} b+(1+\epsilon) L_{i} h \geq L_{i} h \geq C e^{-h_{i}} .
$$

Hence $a_{i} \leq h_{i}$ and so $b_{i} \leq 0$. The claim follows.

Introduce the difference operator $\partial_{j} p=p_{j}-p_{j-1}, j \geq 1$. We shall show that if (2.5) fails then $\liminf _{j \rightarrow+\infty} \partial_{j} a$ is strictly positive, which clearly yields (2.6).

If (2.5) fails, there exists $m \in \mathbb{N}$ and $\epsilon>0$ so that $a_{m}>(1+\epsilon) h_{m}$. Fix $\epsilon$ and assume that $m$ is the minimal positive integer which satisfies this inequality. This means that $a_{j} \leq(1+\epsilon) h_{j}$ for $0 \leq j \leq m-1$.

Consider again the sequence $b_{j}=a_{j}-(1+\epsilon) h_{j}$. By our assumption $b_{m}>0$, and $b_{j} \leq 0$ for $0 \leq j \leq m-1$. Since, as we checked above, this sequence cannot have positive local maxima, we get that $b_{n} \geq b_{n-1}$ for all $n>m$. Take any $n>m$. Then

$$
\partial_{n} a=\partial_{n} b+(1+\epsilon) \partial_{n} h>\partial_{n} h
$$

Furthermore, since

$$
a_{n}=b_{n}+(1+\epsilon) h_{n}>(1+\epsilon) h_{n}
$$


we get

$$
\partial_{n} a-\partial_{n+1} a=L_{n} a \leq C e^{-a_{n}} \leq C e^{-(1+\epsilon) h_{n}} .
$$

Take $N>n$ and observe that in view of (2.8) and (2.9)

$$
\partial_{n} h<\partial_{n} a \leq C e^{-(1+\epsilon) h_{n}}+\partial_{n+1} a \leq \ldots \leq \sum_{j=n}^{N-1} C e^{-(1+\epsilon) h_{j}}+\partial_{N} a .
$$

Since the sequence $\left\{h_{j}\right\}$ is increasing the first term on the right hand side does not exceed

$$
e^{-\epsilon h_{n}} \sum_{j=n}^{N-1} C e^{-h_{j}} \leq e^{-\epsilon h_{n}} \sum_{j=n}^{N-1} L_{j} h=e^{-\epsilon h_{n}}\left(\partial_{n} h-\partial_{N} h\right) \leq e^{-\epsilon h_{n}} \partial_{n} h .
$$

This yields $\partial_{N} a \geq\left(1-e^{-\epsilon h_{n}}\right) \partial_{n} h$. Fix $n$ large enough so that $1-e^{-\epsilon h_{n}} \geq 1 / 2$. Letting $N \rightarrow+\infty$, we obtain that

$$
\liminf _{N \rightarrow+\infty} \partial_{N} a \geq \frac{1}{2} \partial_{n} h>0
$$

which yields (2.6).

We complete this section with

\section{Proof of relation (1.2)}

Suppose that $f^{\prime}(\xi)=1$ for each $\xi \in \operatorname{Fix}(f)$. We have to show that $\gamma(f)=1$ which means that

$$
\lim _{n \rightarrow \infty} a_{n}(f) / n=\lim _{n \rightarrow \infty} a_{n}\left(f^{-1}\right) / n=0 .
$$

Put $F(x)=\log f^{\prime}(x)$, and assume on the contrary that

$$
\lim _{n \rightarrow \infty} \max _{x \in[0 ; 1]}\left\{\frac{1}{n} \sum_{i=0}^{n-1} F\left(f^{i}(x)\right)\right\}=\lim _{n \rightarrow \infty} \frac{a_{n}}{n}=c>0 .
$$


Using the Krylov-Bogolyubov argument, we choose a large enough $N$ and a point $y_{(N)}$ such that

$$
\frac{1}{N} \sum_{i=0}^{N-1} F\left(f^{i} y_{(N)}\right)>\frac{c}{2},
$$

and consider a sequence of probability measures on $[0 ; 1]$

$$
\sigma_{N}=\frac{1}{N} \sum_{i=0}^{N-1} \delta_{f^{i} y_{(N)}}
$$

where $\delta_{x}$ is the Dirac measure at $x$. Then there is a subsequence $N_{j} \rightarrow \infty$ such that $\sigma_{N_{j}} \rightarrow \sigma_{\infty}$ (in the weak-* topology), and $\sigma_{\infty}$ is an invariant measure of $f$ such that

$$
\int F d \sigma_{\infty}=\lim _{N_{j} \rightarrow \infty} \int F d \sigma_{N_{j}}>0 .
$$

Note that for every interval $J \subset[0 ; 1] \backslash \operatorname{Fix}(f)$ there is a $k_{0}$ such that $f^{k} J \cap J=$ $\emptyset$ for every $k$ with $|k| \geq k_{0}$. Hence the support of every invariant measure $\sigma$ of $f$ must be contained in the set $\operatorname{Fix}(f)$. Thus $\int F d \sigma_{\infty}=0$ since $F$ vanishes on $\operatorname{Fix}(f)$ due to our assumption. This contradiction proves that $\gamma(f)=1$.

\section{Diffeomorphisms with irregular growth se- quences}

In this section we prove Theorem 1.7. Fix a sequence $\{u(n)\} \in \mathcal{L}$ of positive real numbers, $u(n) \nearrow+\infty$ as $n \rightarrow+\infty$. For a $C^{\infty}$-function $\Delta: \mathbb{R} \rightarrow(0 ;+\infty)$ define recursively a sequence of functions

$$
g_{0}(t)=\frac{\Delta(t+1)}{\Delta(t)}, \quad g_{m+1}(t)=\frac{g_{m}^{\prime}(t)}{\Delta(t)} .
$$

Theorem 3.1 There exists an even $C^{\infty}$-function $\Delta: \mathbb{R} \rightarrow(0 ;+\infty)$ with the following properties:

$$
\int_{-\infty}^{+\infty} \Delta(t) d t<\infty
$$


there is a sequence of positive integers $\tau_{i} \nearrow+\infty$ such that

$$
\sup _{t \in \mathbb{R}} \frac{\Delta\left(t+\tau_{i}\right)}{\Delta(t)} \leq u\left(\tau_{i}\right)
$$

$$
g_{0}(t) \rightarrow 1 \text { and } g_{m}(t) \rightarrow 0 \text { as } t \rightarrow \infty \text { for all integers } m \geq 1
$$

First, we deduce Theorem 1.7. Without loss of generality assume that $\int_{-\infty}^{+\infty} \Delta(t) d t=1$. Put $a(\eta)=\int_{-\infty}^{\eta} \Delta(s) d s$, and define $f:[0 ; 1] \rightarrow[0 ; 1]$ by

$$
f(x)=\left[\begin{array}{ll}
0, & x=0 \\
1, & x=1 \\
a\left(a^{-1}(x)+1\right), & x \in(0 ; 1) .
\end{array}\right.
$$

Proof of Theorem 1.7; Let us verify that $f$ satisfies all the requirements of Theorem 1.7.

1) We claim that $f$ is a $C^{\infty}$-diffeomorphism of the closed interval $[0 ; 1]$, and moreover $f$ is flat at the end points. Indeed, $f$ is continuous on $[0 ; 1]$ and smooth on $(0 ; 1)$. Thus it suffices to check that $f^{\prime}(x) \rightarrow 1$ and $f^{(m)}(x) \rightarrow 0$, $m \geq 2$, as $x \rightarrow 0$ and $x \rightarrow 1$. Put $\eta=a^{-1}(x)$. Then $f^{\prime}(x)=g_{0}(\eta)$,

$$
f^{(2)}(x)=\frac{g_{0}^{\prime}(\eta)}{\Delta(\eta)}=g_{1}(\eta), \ldots, f^{(m+1)}(x)=\frac{g_{m-1}^{\prime}(\eta)}{\Delta(\eta)}=g_{m}(\eta)
$$

for all $m \geq 2$. Here we use that $\frac{d \eta}{d x}=\frac{1}{\Delta(\eta)}$. The claim follows from Property (3.4) of $\Delta$.

2) Note that $f^{n}(x)=a\left(a^{-1}(x)+n\right)$ for all $n \in \mathbb{Z}$. Hence

$$
\Gamma_{n}(f)=\max \left(\max _{x} \frac{a^{\prime}\left(a^{-1}(x)+n\right)}{a^{\prime}\left(a^{-1}(x)\right)}, \max _{x} \frac{a^{\prime}\left(a^{-1}(x)-n\right)}{a^{\prime}\left(a^{-1}(x)\right)}\right)=\sup _{\eta \in \mathbb{R}} \frac{\Delta(\eta+n)}{\Delta(\eta)}
$$

since $\Delta$ is even. Property (3.3) guarantees that $\Gamma_{\tau_{i}}(f) \leq u\left(\tau_{i}\right)$. This completes the proof. 
It remains to prove Theorem 3.1 , that is to construct an even $C^{\infty}$-function with properties (3.2)-(3.4).

Idea of the construction: As the first approximation for $\Delta$ we start with an even $C^{\infty}$-function $h: \mathbb{R} \rightarrow(0 ;+\infty)$ satisfying conditions (3.2) and (3.4) and such that $h(0)=1$, and $h(t)$ decreases for $t>0$. Consider the weighted average

$$
\left(A_{\tau, \mu} h\right)(t)=\sum_{j \in \mathbb{Z}} \mu^{|j|} h(t+j \tau)
$$

where $0<\mu<1$. It is not difficult to check that

$$
\mu \leq \sup _{t \in \mathbb{R}} \frac{\left(A_{\tau, \mu} h\right)(t+\tau)}{\left(A_{\tau, \mu} h\right)(t)} \leq \frac{1}{\mu},
$$

since the average produces "self-similar humps" of relative magnitude $\mu$. Then iterating this procedure with properly chosen sequences $\tau_{i} \rightarrow \infty$ and $\mu_{i} \rightarrow 0$, we get an even function satisfying conditions (3.2) and (3.3). Unfortunately, we loose the smoothness property (3.4).

To mend this, we modify the operator $A$ by introducing an additional rescaling:

$$
\sum_{j \in \mathbb{Z}} \mu^{|j|} h\left(\alpha_{j}(t+j \tau)\right)
$$

where $\alpha_{j}$ are suitably chosen rescaling factors. The new average still produces self-similar humps, this time without spoiling the behaviour of the derivatives. Then an infinite repetition of this process (with a careful choice of values of $\tau, \mu$ and $\left\{\alpha_{j}\right\}$ in each step) does the job.

Formal construction: Let $h$ be an even $C^{\infty}$-function $h: \mathbb{R} \rightarrow(0 ;+\infty)$ such that $h(0)=1, h(t)$ decreases for $t>0$ and $h(t)=\frac{1}{t \log ^{2} t}$ for $t \geq 3$. Take a function $\tau: \mathbb{N} \rightarrow \mathbb{N}$ such that

$$
\sum_{i \in \mathbb{N}} \frac{1}{\log u\left(\tau_{i}\right)}<\infty ;
$$


We also assume that the value $\tau_{1}$ is sufficiently large. Define now two functions $\mu: \mathbb{N} \rightarrow(0 ; 1)$ and $\gamma: \mathbb{N} \times \mathbb{Z} \rightarrow(1 ;+\infty)$ as follows:

$$
\begin{aligned}
& \mu_{i}=u^{-1 / 4}\left(\tau_{i}\right), \\
& \gamma_{i, \ell}=\min \left(\left|\log \mu_{i}\right|, \mu_{i}^{-\frac{1}{\sqrt{|\ell|}}}\right) \quad \text { for } \quad \ell \neq 0, \\
& \text { and } \gamma_{i, 0}=\left|\log \mu_{i}\right| .
\end{aligned}
$$

Let $\mathbb{Z}^{\infty}$ be the space of all functions $k: \mathbb{N} \rightarrow \mathbb{Z}$ with finitely many non-zero values $k_{i}$. Define two functions on $\mathbb{Z}^{\infty}$ as follows:

$$
\varphi(k)=\prod_{i \in \mathbb{N}} \mu_{i}^{\left|k_{i}\right|}, \quad \theta(k)=\prod_{i \in \mathbb{N}} \gamma_{i, k_{i}}^{\left|k_{i}\right|}
$$

(the products have only finitely many factors). Mention that the functions $\varphi$ and $\varphi \theta$ are bounded by one:

$$
\varphi(k) \leq \varphi(k) \theta(k) \leq \prod_{i \in \mathbb{N}} \mu_{i}^{\left|k_{i}\right|-\sqrt{\left|k_{i}\right|}} \leq 1 .
$$

Fundamental definition: Put

$$
\Delta(t)=\sum_{k \in \mathbb{Z}^{\infty}} \varphi(k) h(\varphi(k) \theta(k)(t-\langle k, \tau\rangle)),
$$

where $\langle k, \tau\rangle=\sum_{i \in \mathbb{N}} k_{i} \tau_{i}$.

The function $\Delta$ is well defined since

$$
\sum_{k \in \mathbb{Z} \infty} \varphi(k)=\prod_{i \in \mathbb{N}} \sum_{j \in \mathbb{Z}} \mu_{i}^{|j|}=\prod_{i \in \mathbb{N}} \frac{1+\mu_{i}}{1-\mu_{i}} .
$$

The latter product is convergent since

$$
\sum_{i \in \mathbb{N}} \mu_{i}=\sum_{i \in \mathbb{N}} u^{-1 / 4}\left(\tau_{i}\right)<\infty
$$


in view of (3.5). Since the function $k \mapsto \varphi(k) \theta(k)$ is bounded on $\mathbb{Z}^{\infty}$ and since all derivatives $h^{(m)}$ are bounded, the same argument shows that $\Delta$ is a $C^{\infty}$-function with

$$
\Delta^{(m)}(t)=\sum_{k \in \mathbb{Z}^{\infty}} \varphi^{m+1}(k) \theta^{m}(k) h^{(m)}(\varphi(k) \theta(k)(t-\langle k, \tau\rangle)) .
$$

Clearly, the function $\Delta$ is even. We have to show that it satisfies conditions (3.2)-(3.4).

Convergence of the integral (3.2): Since the function $h$ is integrable, it suffices to check convergence of the series

$$
\sum_{k \in \mathbb{Z}^{\infty}} \frac{1}{\theta(k)}=\prod_{i \in \mathbb{N}} \sum_{\ell \in \mathbb{Z}} \frac{1}{\gamma_{i, \ell}^{\ell \ell}} \leq \prod_{i \in \mathbb{N}}\left\{1+2 \sum_{\ell \geq 1}\left|\log \mu_{i}\right|^{\ell \ell}+2 \sum_{\ell \geq 1} \mu_{i}^{\sqrt{\ell}}\right\} .
$$

Since

$$
\sum_{\ell \geq 1} \mu^{\sqrt{\ell}}<\mu+\int_{1}^{+\infty} \mu^{\sqrt{\ell}} d \ell=O\left(\frac{1}{|\log \mu|^{2}}\right),
$$

for $\mu \rightarrow 0$, the right-hand side of the previous expression is bounded by $\prod_{i \in \mathbb{N}}\left\{1+\right.$ const $\left.\left|\log \mu_{i}\right|^{-1}\right\}$. But this product is finite in view of (3.5).

Proof of (3.3): Denote by $e^{i} \in \mathbb{Z}^{\infty}$ the vector with $e_{n}^{i}=\delta_{i n}$, where $i, n \in \mathbb{N}$. We have

$$
\begin{aligned}
\Delta\left(t+\tau_{i}\right) & =\sum_{k \in \mathbb{Z}^{\infty}} \varphi(k) h\left(\varphi(k) \theta(k)\left(t-\left\langle k-e^{i}, \tau\right\rangle\right)\right) \\
& =\sum_{k \in \mathbb{Z}^{\infty}} \varphi\left(k+e^{i}\right) h\left(\varphi\left(k+e^{i}\right) \theta\left(k+e^{i}\right)(t-\langle k, \tau\rangle)\right) .
\end{aligned}
$$

Comparing this with the definition of $\Delta(t)$, we get that

$$
\frac{\Delta\left(t+\tau_{i}\right)}{\Delta(t)} \leq \sup _{k \in \mathbb{Z}^{\infty}} \frac{\varphi\left(k+e^{i}\right)}{\varphi(k)} \cdot \sup _{k \in \mathbb{Z}^{\infty}} \sup _{s \in \mathbb{R}} \frac{h(s)}{h\left(c_{k} s\right)},
$$

where

$$
c_{k}=\frac{\varphi(k) \theta(k)}{\varphi\left(k+e^{i}\right) \theta\left(k+e^{i}\right)} .
$$




\section{Lemma 3.7}

$$
\mu_{i} \leq \frac{\varphi\left(k+e^{i}\right)}{\varphi(k)} \leq \frac{1}{\mu_{i}} \quad \text { and } \quad \mu_{i} \leq \frac{\theta\left(k+e^{i}\right)}{\theta(k)} \leq \frac{1}{\mu_{i}}
$$

for all $k \in \mathbb{Z}^{\infty}, i \in \mathbb{N}$.

Assume the lemma and note that

$$
\sup _{s \in \mathbb{R}} \frac{h(s)}{h(c s)} \leq 1 \quad \text { if } 0<c \leq 1
$$

and

$$
\sup _{s \in \mathbb{R}} \frac{h(s)}{h(c s)} \leq \text { const } c\left(1+\log ^{2} c\right) \quad \text { if } c>1 .
$$

It follows from Lemma 3.7 that $c_{k} \leq \mu_{i}^{-2}$, and hence

$$
\sup _{s \in \mathbb{R}} \frac{h(s)}{h\left(c_{k} s\right)} \leq \mathrm{const} \cdot\left(\frac{1}{\mu_{i}^{2}}\right)\left(1+4 \log ^{2} \frac{1}{\mu_{i}}\right) \text {. }
$$

Since $\mu_{i}^{-1}=u\left(\tau_{i}\right)^{1 / 4} \geq u\left(\tau_{1}\right)^{1 / 4}$, we conclude that

$$
\sup _{s \in \mathbb{R}} \frac{h(s)}{h\left(c_{k} s\right)} \leq \frac{1}{\mu_{i}^{3}}
$$

provided $\tau_{1}$ is sufficiently large. Applying again Lemma 3.7 and substituting the last inequality into (3.6) we conclude that

$$
\frac{\Delta\left(t+\tau_{i}\right)}{\Delta(t)} \leq \frac{1}{\mu_{i}} \cdot \frac{1}{\mu_{i}^{3}}=u\left(\tau_{i}\right)
$$

which proves (3.3) modulo the lemma.

Proof of Lemma 3.7; The first inequality follows from the fact that

$$
\frac{\varphi\left(k+e^{i}\right)}{\varphi(k)}=\mu_{i}^{\left|k_{i}+1\right|-\left|k_{i}\right|}=\mu_{i}^{ \pm 1} .
$$

For the second one, we put $\alpha_{i, \ell}=\left|\log \mu_{i}\right|^{|\ell|}$ and $\beta_{i, \ell}=\mu_{i}^{-\sqrt{|\ell|}}$ and notice that

$$
\frac{\theta\left(k+e^{i}\right)}{\theta(k)}=\frac{\min \left(\alpha_{i, k_{i}+1}, \beta_{i, k_{i}+1}\right)}{\min \left(\alpha_{i, k_{i}}, \beta_{i, k_{i}}\right)} .
$$


Further,

$$
\begin{aligned}
& \frac{\alpha_{i, \ell+1}}{\alpha_{i, \ell}}=\left|\log \mu_{i}\right|^{ \pm 1} \in\left[\mu_{i}, \frac{1}{\mu_{i}}\right], \\
& \frac{\beta_{i, \ell+1}}{\beta_{i, \ell}} \leq \sup _{s \geq 0}\left(\frac{1}{\mu_{i}}\right)^{\sqrt{s+1}-\sqrt{s}}=\frac{1}{\mu_{i}},
\end{aligned}
$$

and

$$
\frac{\beta_{i, \ell+1}}{\beta_{i, \ell}} \geq \inf _{s \geq 0} \mu_{i}^{\sqrt{s+1}-\sqrt{s}}=\mu_{i}
$$

Note now that for every 4 positive numbers $a, b, c, d$

$$
\min \left(\frac{a}{c}, \frac{b}{d}\right) \leq \frac{\min (a, b)}{\min (c, d)} \leq \max \left(\frac{a}{c}, \frac{b}{d}\right) .
$$

Applying this to (3.8) and using (3.9)-(3.11), we conclude that

$$
\mu_{i} \leq \frac{\theta\left(k+e^{i}\right)}{\theta(k)} \leq \frac{1}{\mu_{i}}
$$

This proves the lemma.

It remains to check that the function $\Delta$ satisfies the asymptotic regularity condition (3.4). We start with

Preliminary estimates: the function $\Delta(t)$ satisfies conditions

$$
\sup _{|s| \leq 1} \sup _{t \in \mathbb{R}} \frac{\Delta(t+s)}{\Delta(t)}<\infty,
$$

and

$$
\lim _{t \rightarrow \infty} \Delta(t)=0
$$

Estimate (3.12) holds for $h$, and therefore for $\Delta$ since the function $k \rightarrow$ $\varphi(k) \theta(k)$ is bounded on $\mathbb{Z}^{\infty}$. Then (13.13) follows from integrability of $\Delta$ (see (3.2) ), and (3.12).

The next lemma shows that we did not loose much in the asymptotic regularity of $\Delta$ compared with that of $h$. 
Lemma 3.14 For every $m \in \mathbb{N}$ and every $c \in[0 ; 1)$,

$$
\lim _{t \rightarrow \infty} \frac{\max _{[t ; t+1]}\left|\Delta^{(m)}\right|}{\Delta^{m+c}(t)}=0 .
$$

Proof: We show that for every $m \in \mathbb{N}$ and every $c \in[0 ; 1)$

$$
\lim _{t \rightarrow \infty} \frac{\Delta^{(m)}}{\Delta^{m+c}}(t)=0 .
$$

Together with (3.12) this yields the lemma.

In view of (3.13) it suffices to show that the function $\Delta^{(m)} / \Delta^{m+c}$ is bounded on $\mathbb{R}$ for every $m \in \mathbb{N}$ and $c \in[0 ; 1)$. Fix such $m$ and $c$. It is easy to see by induction in $m$ that

$$
\left(\frac{1}{t \log ^{2} t}\right)^{(m)}=\frac{(-1)^{m} m !}{t^{m+1} \log ^{2} t}+\Lambda_{m}(t), \quad m \geq 0,
$$

where $\Lambda_{m}$ is a linear combination of the functions $\frac{1}{t^{m+1} \log ^{k} t}$ with $3 \leq k \leq$ $m+2$. Therefore,

$$
h^{(m)}(t)=\frac{(-1)^{m} m !(1+o(1))}{t^{m+1} \log ^{2} t}, \quad t \rightarrow+\infty,
$$

for each $m \geq 0$, and the function $t \mapsto h^{(m)}(t) / h^{m+c}(t)$ is bounded. Then we have

$$
\left|\Delta^{(m)}(t)\right| \leq \kappa \sum_{k \in \mathbb{Z}^{\infty}} \varphi^{m+1}(k) \theta^{m}(k) h^{m+c}\left(s_{k}\right),
$$

where $s_{k}=\varphi(k) \theta(k)(t-\langle k, \tau\rangle)$, and

$$
\kappa=\kappa_{m, c}=\sup _{t \in \mathbb{R}} \frac{\left|h^{(m)}(t)\right|}{h^{m+c}(t)} .
$$

We claim that

$$
\nu_{m, c}:=\sup _{k \in \mathbb{Z}^{\infty}} \varphi^{1-c}(k) \theta^{m}(k)<\infty .
$$


Combining the claim with the elementary inequality

$$
\sum_{i} x_{i}^{r} \leq\left(\sum_{i} x_{i}\right)^{r}, r \geq 1,0 \leq x_{i} \leq 1,
$$

we readily complete the proof of (3.12):

$$
\begin{aligned}
& \left|\Delta^{(m)}(t)\right| \leq \kappa \nu_{m, c} \sum_{k \in \mathbb{Z}^{\infty}} \varphi^{m+c}(k) h^{m+c}\left(s_{k}\right) \\
& \leq \kappa \nu_{m, c}\left(\sum_{k \in \mathbb{Z}^{\infty}} \varphi(k) h\left(s_{k}\right)\right)^{m+c}=\kappa \nu_{m, c} \Delta(t)^{m+c} .
\end{aligned}
$$

To prove (3.16), we set

$$
K(m, c)=\left\{k \in \mathbb{Z}^{\infty}: \varphi^{1-c}(k) \theta^{m}(k) \leq 1\right\}
$$

and check that the complement $\mathbb{Z}^{\infty} \backslash K(m, c)$ is a finite set. Indeed, if $k \in$ $\mathbb{Z}^{\infty} \backslash K(m, c)$, then

$$
\prod_{i \in \mathbb{N}}\left(\mu_{i}^{1-c} \gamma_{i, k_{i}}^{m}\right)^{\left|k_{i}\right|}=\varphi^{1-c}(k) \theta^{m}(k)>1,
$$

and therefore at least one of the factors on the left-hand side is bigger than one. Hence there exists $i \in \mathbb{N}$ such that

$$
\mu_{i}^{1-c} \gamma_{i, k_{i}}^{m} \geq 1
$$

which is equivalent to two inequalities:

$$
\mu_{i}^{1-c}\left|\log \mu_{i}\right|^{m} \geq 1 \quad \text { and } \quad \mu_{i}^{1-c-m / \sqrt{\left|k_{i}\right|}} \geq 1
$$

The first inequality shows that

$$
\frac{\left|\log \mu_{i}\right|}{\log \left|\log \mu_{i}\right|} \leq \frac{m}{1-c},
$$

therefore there exists a number $j(m, c)$ such that $i \leq j(m, c)$. The second inequality tells us that

$$
\left|k_{i}\right| \leq\left(\frac{m}{1-c}\right)^{2}
$$


Hence

$$
\#\left(\mathbb{Z}^{\infty} \backslash K(m, c)\right) \leq\left(2\left(\frac{m}{1-c}\right)^{2}+1\right) j(m, c),
$$

and (3.16) follows. The lemma is proved.

Verification of condition (3.4): For a function $v: \mathbb{R} \rightarrow \mathbb{R}$ denote $(\omega v)(t)=v(t+1)-v(t)$. Recall that we are proving Property (3.4) which deals with functions $g_{m}$ where

$$
g_{0}(t)=\frac{\Delta(t+1)}{\Delta(t)}=\frac{(\omega \Delta)(t)}{\Delta(t)}+1 \quad \text { and } \quad g_{m+1}(t)=\frac{g_{m}^{\prime}(t)}{\Delta(t)} .
$$

First, note that

$$
g_{0}(t)-1=\frac{\Delta(t+1)-\Delta(t)}{\Delta(t)}=\frac{\Delta^{\prime}\left(x_{t}\right)}{\Delta(t)}
$$

for some $x_{t} \in[t, t+1]$. Then Lemma 3.14 yields $g_{0}(t) \rightarrow 1$ as $t \rightarrow \infty$. It remains to show that $g_{m}(t) \rightarrow 0$ as $t \rightarrow \pm \infty$ for every $m \geq 1$.

Lemma 3.17 The function $g_{m}$ is a finite linear combination of functions of the form

$$
R=\frac{\omega \Delta^{(p)}\left(\Delta^{\prime}\right)^{\ell_{1}} \cdots\left(\Delta^{(m-1)}\right)^{\ell_{m-1}}}{\Delta^{\ell}}
$$

where $p, \ell_{1}, \ldots, \ell_{m-1} \geq 0$ and

$$
2 \ell_{1}+\cdots+m \ell_{m-1}+p+2>\ell .
$$

Proof: We use induction in $m$. For $m=0$ we have $p=0, \ell=1, \ell_{1}=$ $\cdots=\ell_{m-1}=0$. Inequality $(3.18)_{0}$ reads $2>1$. Assume the statement of the lemma for $m$, and prove it for $m+1$. Note that $g_{m+1}$ is a finite linear combination of functions of the form $R^{\prime} / \Delta$. In turn, $R^{\prime} / \Delta$ is a linear combination of the following expressions:

$$
\frac{\omega \Delta^{(p+1)}\left(\Delta^{\prime}\right)^{\ell_{1}} \cdots\left(\Delta^{(m-1)}\right)^{\ell_{m-1}}}{\Delta^{\ell+1}}
$$


$\frac{\omega \Delta^{(p)}\left(\Delta^{\prime}\right)^{\ell_{1}} \cdots\left(\Delta^{(i-1)}\right)^{\ell_{i-1}}\left(\Delta^{(i)}\right)^{\ell_{i}-1}\left(\Delta^{(i+1)}\right)^{\ell_{i+1}+1}\left(\Delta^{(i+2)}\right)^{\ell_{i+2}} \cdots\left(\Delta^{(m-1)}\right)^{\ell_{m-1}}}{\Delta^{\ell+1}}$,

where $i=1, \ldots, m-1$, and

$$
\frac{\omega \Delta^{(p)}\left(\Delta^{\prime}\right)^{\ell_{1}+1}\left(\Delta^{(2)}\right)^{\ell_{2}} \cdots\left(\Delta^{(m-1)}\right)^{\ell_{m-1}}}{\Delta^{\ell+2}} .
$$

Let us check (3.18) $)_{m+1}$ in each of these 3 cases using $(3.18)_{m}$ :

- $\ell+1<p+1+2+2 \ell_{1}+\cdots+m \ell_{m-1}$;

- $\ell+1<p+2+\ell_{1}+\cdots+i \ell_{i-1}+(i+1)\left(\ell_{i}-1\right)$

$$
+(i+2)\left(\ell_{i+1}+1\right)+(i+3) \ell_{i+2}+\cdots+m \ell_{m-1}
$$

- $\ell+2<p+2+2\left(\ell_{1}+1\right)+3 \ell_{2}+\cdots+m \ell_{m-1}$.

This completes the proof.

Now we are ready to finish the proof of (3.4). It suffices to show that $R(t) \rightarrow 0$ as $t \rightarrow \infty$, where $R$ is defined in Lemma 3.17. Write

$$
2 \ell_{1}+\cdots+m \ell_{m-1}+p+2=\ell+1+r
$$

with $r \geq 0$. Choose numbers $\delta_{0}, \ldots, \delta_{m-1}$ so that $\delta_{i} \in[0 ; 1)$ and

$$
\left(1-\delta_{0}\right)+\ell_{1}\left(1-\delta_{1}\right)+\cdots+\ell_{m-1}\left(1-\delta_{m-1}\right)=1 .
$$

Then

$$
\ell=\left(p+1+\delta_{0}\right)+\ell_{1}\left(1+\delta_{1}\right)+\ell_{2}\left(2+\delta_{2}\right)+\cdots+\ell_{m-1}\left(m-1+\delta_{m-1}\right)-r .
$$

Rewrite $R$ as follows:

$$
R=\frac{\omega \Delta^{(p)}}{\Delta^{p+1+\delta_{0}}} \cdot\left(\frac{\Delta^{\prime}}{\Delta^{1+\delta_{1}}}\right)^{\ell_{1}} \cdots \cdot\left(\frac{\Delta^{(m-1)}}{\Delta^{m-1+\delta_{m-1}}}\right)^{\ell_{m-1}} \cdot \Delta^{r} .
$$

Then by Lemma 3.14 and by (3.13), $R(t) \rightarrow 0$ as $t \rightarrow \infty$. This completes the proof of (3.4), and therefore finishes off the proof of Theorem 3.1 


\section{Acknowledgments}

Fëdor Nazarov generously helped us with the first version of the growth lemma. In the present form, it appeared after discussions with Lennart Carleson and Alexei Poltoratskii. Amir Hadadi (an undergraduate student at Tel Aviv) showed us a piecewise constant function $\Delta$ satisfying (3.2) and a version of (3.3). Jean-Christophe Yoccoz sketched an example of a diffeomorphism of finite smoothness whose growth is slower than the linear one along a suitable subsequence. We thank all of them, as well as Alexander Borichev, Pierre de la Harpe, Anatole Katok, Patrice Le Calvez and Felix Schlenk for useful discussions and the referee for helpful critical remarks.

Part of this work was done during the stay of the second named author at the Mittag-Leffler Institute of the Royal Swedish Academy of Sciences in the Winter 2002. He thanks this institute for the kind hospitality.

\section{References}

[B] A. BonICHEv, The distortion growth for iterations of diffeomorphisms of the interval, Preprint 2003.

[DG] G. D'Ambra and M. Gromov, Lectures on transformation groups: geometry and dynamics, in Surveys in Differential Geometry, supplement to the J. Diff. Geom., number 1, 1991, pp. $19-112$.

[K] H. KaWABE, On the conjugation of local diffeomorphisms infinitely tangent to the identity, in "Foliations", Advanced Studies in Pure Math. 5, 1985, pp. 461-481.

[LMR] A. Lubotzky, S. Mozes and M.S. Raghunathan, The word and riemannian metrics on lattices in semisimple Lie groups, IHES Publ. Math. 91 (2000), 5-53. 
[P1] L. Polterovich, Growth of maps, distortion in groups and symplectic geometry, Invent. Math. 150 (2002), 655 - 686.

[P2] L. Polterovich, Slow symplectic maps, continued fractions and related stories, in "Symplectic and Contact Topology: Interactions and Perspectives", Y. Eliashberg, B. Khesin and F. Lalonde eds., Fields Institute Communications, Vol. 35, AMS, 2003.

[PSib] L. Polterovich, K.F. Siburg, On the asymptotic geometry of area-preserving maps, Math. Res. Letters 7 (2000), 233 -243.

[PS] L. Polterovich and J.-C. Sikorav, A linear isoperimetric inequality for the punctured Euclidean plane, preprint math. GR/0106216.

[Se] F. Sergeraert, Feuilletages et difféomorphismes infiniment tangents à l'identité, Invent. Math. 39 (1977), 253-275.

[T] F. TAKens, Normal forms for certain singularities of vector fields, Ann. Inst. Fourier 23 (1973), 163-195. 